\title{
Study on Internet of Things application for High- speed Train Maintenance, Repair and Operation (MRO)
}

\author{
Weijiao ZHANG \\ Institute of Computing Technology \\ China Academy of Railway Sciences \\ Beijing, China \\ Sunpercher@163.com
}

\begin{abstract}
EMU IoT-system, which is oriented to the MRO of high-speed trains, was studied with implementations of sensing technologies like RFID, sensors, etc; with regarding to the future requirements of safety, orderly, efficient and high-quality in EMU maintenance service of China. In operation: massive seamless embedded RFID tags and sensors, on the basis of trainground transmission network, perceive running and defects status of high-speed train in real-time; realizing holographic train visualization and transit alerts. In maintenance and repair: multisource and multilevel raw data, from the collection of various production aspects such as trains flow, parts flow, labor jobs flow and equipments, monitor productive process and logistics of train maintenance in total life cycle. The general framework and detailed technical solution were presented in this paper; for the efficient processing of raw data, a semantic analysis measure driven by complex event mechanism was introduced; finally, a quantitative evaluation of productivity upgrading owing to IoT was made. All RFID applications involved are running or experienced pilot tests in factory environment.
\end{abstract}

Keywords-intelligent transportation; internet of things (IoT); high-speed trian; semantic analysis; complex event processing

\section{INTRODUCTION}

EMU maintenance is essentially a routine procedure which improves the internal and external states as well as service conditions of high-speed train through labor or automatic devices. Operation-level maintenance mainly concerns the routine examination or focused inspection; whereas overhaullevel maintenance engages in part-level mending, whose processes include dismantle, repair and assembly. Workshop personnel are labor subjects of production, trains are manufactured objects, and maintenance facilities are productive tools.

High-speed train mending is characterized by highly specialization, routinization and intensification, involving complicated inter-organizational and multi-professional cooperation among EMU maintenance base. EMU Parts can be divided into examined ones and spare ones according to origins; examined parts were maintenance objects which came from trains, while spare (interchangeable) parts are necessary resource for maintenance which were supplied by materials center.
EMU Maintenance involves sophisticated managements of discrete production process (or WIP, work-in-process), and logistics supply chain, etc. At present, there are plenty of applications with regarding to the utilization of IOT technologies. Lufthansa airline applied RFID to airplane maintenance, along with the tracking of perishable and dangerous articles; a few clothing companies introduced IoT technologies to production-line, replacing traditional barcode work tickets with RFID tags, while identifying staffs by smart cards; in the fields of automobile, valuable sophisticated devices manufacturing, IoT has been utilized to different degrees. The Chinese ministry of railways is managing to develop intelligent high-speed trains by the way of integrating massive sensors and RFID tags to every key parts of highspeed train, implementing holographic information perception. This study is expected to increase productive output of EMU maintenance to $25 \%$ for the operation and $20 \%$ for the overhaul respectively.

\section{TECHNICAL SOLUTION OF APPLICATION}

\section{A. Parts logistics flow}

Parts logistics is the core of EMU maintenance activity, which involves examined parts flowing within workshops and spare parts delivering from warehouse. Maintenance tasks are orderly organically executed among workshops in EMU base according to a well-defined process flow. EMU is made up of over 40,000 parts; while materials center, workshop warehouse, and tool rooms manage swap spare parts, consumption articles and maintenance tools in a concentrated way, simultaneously supporting timely supply, safety stock, and source tracing; realtime monitoring of valuable parts, dangerous and perishable articles are also taken during inventory period, tracking location and status information of the key articles frequently.

EMU Parts adopts a metal, liquid and heat resistant fashioned RFID tags, whose carrier frequency works at $868 \sim 928 \mathrm{MHz}$, and as well characterized by adhesive, small size and low price; communication protocol follows international standards EPCglobal Class1 Gen2 and ISO18000$6 c$; according to actual conditions, reading distance are adjusted through altering reader transmitted power and antenna gain; for the need of indoor key objects location, it is also compatible with RTLS international standards ISO/IEC 24730 series. 


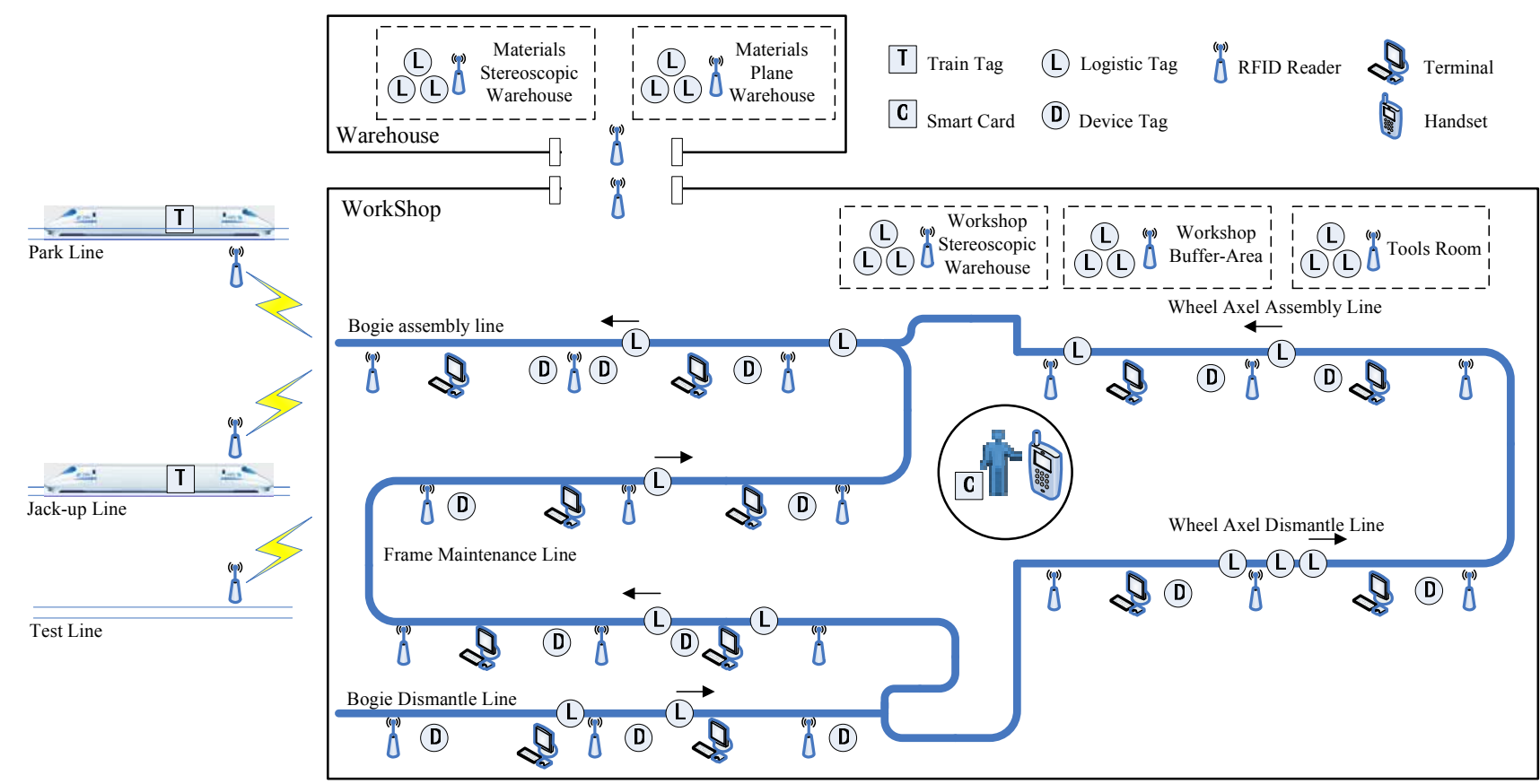

Figure1. IoT Topology of EMU maintenance, repair and overhaul

\section{B. Labor jobs flow}

Smart cards facilitate human authentication through information system, concerning a large class of activities like work performance confirmation, device rights certification, software access control, attendance management, catering, etc. Smart cards and their readers adopt unified standards, storage format and encryption protocol; and were managed by information system in centralized mode. Smart cards are PVC packaged and ISO7816 compatible, whose RFID microchip complies with ISO/IEC 14443 Type A and work at HF frequency range $(13.56 \mathrm{MHz})$, implementing non-contact identification ranged from 0 to $10 \mathrm{~cm}$.

\section{Trains flow}

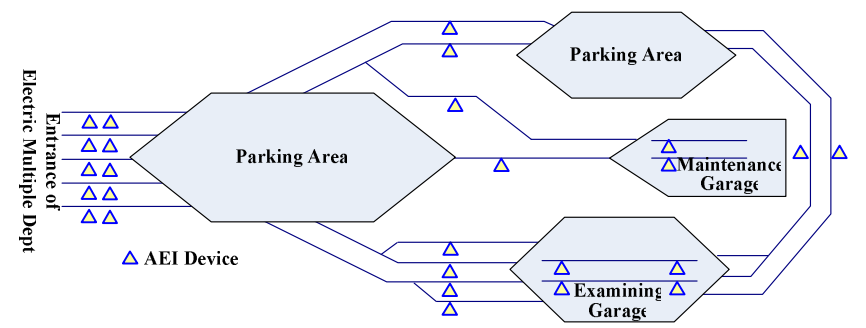

Figure2. AEI layout of EMU maintenance base

As early as 2001, RFID has been maturely applied to automatic running train identification, as shown in Fig. 2. AEI devices are placed on passage ways, throat areas, or intersection areas between concentrated and non-concentrated stop lines at electric multiple dept. The information collected by AEI is consist of train number, train type, marshalling, etc; along with the train route controlling supported by computer interlocking system, the centralized traffic control of arrival, shunting and departure among rail tracks are realized. Trains are equipped with UHF passive RFID tags, whose working frequency is $902 \mathrm{MHz} \sim 928 \mathrm{MHz}$ with reading distance as far as $10 \mathrm{~m}$, and being compatible with Chinese railway industry standard TB/T3070-2002.

\section{Equipment and facility assets}

In pace with the extensively integration of high technologies in EMU manufacturing, the amount, kinds, value and technical level of maintenance equipments in maintenance organization has been greatly improved. By the adoption of device RFID tags and handset reader, the information managements of point inspection, regular examination, and failure report were realized. Due to the directional reading fashion of handset, mass objects identification from long distance doesn't take place in such a scenario. The RFID tags work at $13.56 \mathrm{MHz}$, and are compatible to ISO/IEC 14443 with reading distance of $10 \mathrm{~cm}$.

\section{PRODUCTIVE DATA PROCESSING}

\section{A. Event-driven architecture of IoT system}

Owing to the extensive adoption of RFID, sensors, location technologies; when EMU base conducts maintenance activities, a vast amount of productive raw data is generated within short time. While the warehouse in/out, the spare parts delivery for production, or the examined parts flowing through each process workstation at production-line orderly; RFID tags and smart cards are identified by RFID readers placed in specific locations. While trains moving and shunting among different railway tracks, the RFID tags fixed in engine cars are continuously read with identification information. While a condition based process is executed, the state of examined part is detected by sensors or other automatic devices in order to determine later processes. 
During all the data acquisition modes as described above, three kinds of information owned by objects are collected; they are attribute, state, and location. By which the goal of internet of things, i.e. the ubiquitous connections of any things anywhere, is realized.

However, collected data of EMU maintenance is usually lack of semantic interrelationship; complex event processing (CEP) is a way of preprocessing raw data, it aggregates simple events into complex event efficiently, and is widely used in information system.

Event is defined as a set of significant changes and includes simple events and complex events. Simple event is atomic event which happened at a moment; while complex event is made up of simple or complex events which happened at a moment and may also well be in a period.

Simple event can be formal expressed as Eq. (1):

$$
E_{s}(t)=e(a, t)
$$

Complex event can be expressed as Eq. (2) or Eq. (3) depending on it happened at a moment or in a period.

$$
\begin{gathered}
E_{c}(t)=e(a, e, t) \\
E_{c}(t)=e\left(a, e, t_{1}, t_{2}\right)
\end{gathered}
$$

In the above equations, $a$ is attribute list, $e$ is event list, and $t$ is the time of the event; with respect of EMU IoTsystem, $a=(o, p, s)$, o is the physical object being monitored, $\mathrm{p}$ is the position where event happened, and $\mathrm{s}$ is the concerned states of the object.

Several event operators are defined for complex events aggregation: ' $\wedge$ ', ' $\vee$ ', ' $\neg$ ' represent the logical relationship of events and mean 'and', 'or', 'not' respectively; ';' is used to connect several events which happened order by a sequence 'Within $\left(E(t), T_{1}, T_{2}\right)$ ' represents that event $E(t)$ happened between $T_{1}$ and $T_{2}$.

\section{B. Definition of simple RFID-read event}

RFID-read event is the simplest event of EMU IoT-system; as event position $p$ can be roughly estimated by the location of RFID reader, and RFID device does not handle state data, RFID-read event is simplified into Eq. (4):

$$
E_{r}(t)=e(o, p, t)
$$

\section{Complex enents of EMU maintenance}

\section{1) Warehouse in/out}

Warehouse-in and warehouse-out events happened when materials (especially spare parts) import or export through warehouse door. RFID readers placed at dock door and cargo shelf scan near RFID tags frequently, therefore the event of warehouse-in and warehouse-out are represented as Eq. (5) and Eq. (6) respectively:

$$
\begin{aligned}
& E_{1}(t)=\operatorname{Within}\left(e\left(o_{L}, p_{I}, t^{\prime}\right) ; \neg e\left(o_{L}, p_{I}, t^{\prime \prime}\right), T_{1}, T_{2}\right) \\
& E_{2}(t)=\operatorname{Within}\left(\neg e\left(o_{L}, p_{I}, t^{\prime}\right) ; e\left(o_{L}, p_{I}, t^{\prime \prime}\right), T_{1}, T_{2}\right)
\end{aligned}
$$

$o_{L}$ represents logistics object, $p_{I}$ represents inventory place, and $t^{\prime}<t^{\prime \prime}, T_{1}<T_{2}, t^{\prime}, t^{\prime \prime} \in\left(T_{1}, T_{2}\right)$.

\section{2) Rail track shunting}

Rail track shunting event happened when train shunting from one track to another, it's formal described is as shown as Eq. (7):

$$
E_{3}(t)=\text { Within }\left(e\left(o_{T}, p_{a}, t^{\prime}\right) ; e\left(o_{T}, p_{b}, t^{\prime \prime}\right), T_{1}, T_{2}\right)
$$

$o_{T}$ represents a electric multiple units whose engine car is installed with RFID tag, $p_{a}, p_{b}$ is rail track; train shunted from $p_{a}$ to $p_{b}$.

\section{3) Spare parts delivery}

When spare parts were transported to the maintenance workstations at production line, the production delivery event happened as shown as Eq. (8), in which $p_{w}$ means a workshop place:

$$
E_{4}(t)=\text { Within }\left(\neg e\left(o_{L}, p_{W}, t^{\prime}\right) ; e\left(o_{L}, p_{W}, t^{\prime \prime}\right), T_{1}, T_{2}\right)
$$

4) Maintenance or quality examine process

When maintenance or quality examining processes executed at workshop, the actions of worker card swiping and examined parts identification happened simultaneously as shown as Eq. (9) and Eq. (10):

$$
\begin{gathered}
E_{5}(t)=\operatorname{Within}\left(\neg e\left(o_{C}, p_{W}, t_{C}^{\prime}\right) ; e\left(o_{C}, p_{W}, t_{C}^{\prime \prime}\right), T_{1}, T_{2}\right) \\
E_{6}(t)=\operatorname{Within}\left(E_{5} \wedge e\left(o_{L}, p_{W}, t_{L}^{\prime}\right)\right)
\end{gathered}
$$

$o_{C}$ represents a smart card, $E_{5}(t)$ is worker card swiping event and $E_{6}(t)$ is a labor job executing event which is defined by the compound of human action and examined object action.

\section{5) Equipments maintaining}

$E_{7}(t)$ is the event of equipments maintaining, in which $o_{D}$ represents device object:

$$
E_{7}(t)=\operatorname{Within}\left(e\left(o_{C}, p_{W}, t_{C}\right) \wedge e\left(o_{D}, p_{W}, t_{D}\right), T_{1}, T_{2}\right)
$$

It's defined by the compound of maintaining crew action and equipment identification action.

6) Repair process according to condition

Some maintenance processes are executed according to the state information which previously collected from predecessor processes. For example, if a wheel is detected as useless, a wheel swapping process should be made. The event formal expressions based on state $a$ and $b$ look like:

$$
\begin{aligned}
& E_{8}^{a}(t)=W i t h i n\left(e\left(o_{L}, p_{W}, s_{a}, t^{\prime}\right) ; E_{6}^{a}\left(t^{\prime \prime}\right), T_{1}, T_{2}\right) \\
& E_{8}^{b}(t)=W i t h i n\left(e\left(o_{L}, p_{W}, s_{b}, t^{\prime}\right) ; E_{6}^{b}\left(t^{\prime \prime}\right), T_{1}, T_{2}\right)
\end{aligned}
$$

\section{THE GENERAL FRAMEWORK}

EMU maintenance oriented Internet of Things can be divided into four layers from the bottom up: 
1) Physical quipment layer: includes physical devices or facilities like RFID reader, smart card, workstation terminal, and etc;

2) Data middleware: as a mechanism which copes with device adaptation, data buffering, and preprocessing of the multi-souce heterogeneous raw data came from equipment layer;

3) Information aggregation layer: convergence and fusion of productive information, it consists of transactional databases, subject oriented data warehouse, and domain metadata model;

4) Application layer: provides versatile application services about logistics flow, labor jobs flow, trains flow, equipment and facility assets; while supporting seamless integration across applications.

With internet of things technologies, the capability of realtime data collection and productive data visualization were greatly enhanced; the information gap between resource planning management and production process control layers was filled; thus the efficiency of productive commands

\begin{tabular}{|c|c|c|c|c|c|c|c|c|}
\hline \multirow{3}{*}{$\begin{array}{l}\text { Application } \\
\text { layer }\end{array}$} & \begin{tabular}{|c} 
Spare parts \\
inventory
\end{tabular} & \begin{tabular}{|c} 
Production \\
line \\
delivery
\end{tabular} & $\begin{array}{c}\text { maintenance } \\
\text { Project } \\
\text { scheduling }\end{array}$ & $\begin{array}{c}\text { Production } \\
\text { process } \\
\text { control }\end{array}$ & \begin{tabular}{|c} 
EMU \\
parking
\end{tabular} & $\begin{array}{c}\text { Equipment } \\
\text { maintaining }\end{array}$ & \begin{tabular}{|} 
Statistic \\
analysis
\end{tabular} \mid & $\begin{array}{c}\text { Management } \\
\text { decision }\end{array}$ \\
\hline & $\begin{array}{r}\text { Logisti } \\
\text { flow } \\
\end{array}$ & & \multicolumn{2}{|c|}{ Labor jobs flow } & \multicolumn{2}{|c|}{ Trains flow } & \multicolumn{2}{|c|}{$\begin{array}{c}\text { Equipment and } \\
\text { facility assets }\end{array}$} \\
\hline & \multicolumn{8}{|c|}{ Enterprise application integration } \\
\hline \multirow{3}{*}{$\begin{array}{l}\text { Information } \\
\text { aggregation } \\
\quad \text { layer }\end{array}$} & \multicolumn{5}{|c|}{ Subject-oriented data warehouse } & \multicolumn{3}{|c|}{ Domain metadata model } \\
\hline & \multicolumn{5}{|c|}{ Transactional database } & \multicolumn{3}{|c|}{ Maintenance workflow model } \\
\hline & \multicolumn{8}{|c|}{ Data resource aggregation } \\
\hline \multirow{6}{*}{$\begin{array}{c}\text { Data } \\
\text { middleware }\end{array}$} & \multicolumn{4}{|c|}{ Data organization and management } & \multirow{2}{*}{\multicolumn{4}{|c|}{ Workload balance }} \\
\hline & \multicolumn{4}{|c|}{ Data compression } & & & & \\
\hline & Data fo & brmatting & and standar & lizing & \multirow{2}{*}{\multicolumn{4}{|c|}{ Data cache and thread pool }} \\
\hline & Dat: & a validatio & $\mathrm{n}$ and clean & & & & & \\
\hline & \multicolumn{8}{|c|}{ Information and communication security } \\
\hline & \multicolumn{8}{|c|}{ Terminal device service } \\
\hline \multirow{2}{*}{$\begin{array}{l}\text { Physical } \\
\text { equipment } \\
\text { layer }\end{array}$} & \multicolumn{3}{|c|}{ RFID Reader } & & Card re & ader & \multirow{2}{*}{\multicolumn{2}{|c|}{$\begin{array}{c}\text { Workstation } \\
\text { terminals } \\
\& \\
\text { handsets }\end{array}$}} \\
\hline & RFID & Tags & Sensors & & Smart & card & & \\
\hline
\end{tabular}

dictation and jobs operation execution was promoted. Data integration and information fusion is a crucial stage of intelligent information processing, with the joint processing of node data from multi-source and multi-level, the completion and richness of high-level business information was greatly improved due to this more accurate and valid inferences came from non-single data source. Based on sufficient physical environment perception, information system realizes deep integration between computational process and physical actions through their interactive feedback communication loops.

\section{PRODUCTIVE EFFECTIVENESS EVALUATION}

Given the large-scale and complexity of EMU maintenance system, in order to evaluate the time efficiency when IoTsystem applied, it's necessary to take a comprehensive investigation of application model, system logs and technical specifications with reasonable assumption. The application modes $\Omega$ include four aspects: trains flow $\mathrm{T}$, parts logistics flow $\mathrm{L}$, labor jobs flow $\mathrm{C}$, equipment and facility assets $\mathrm{D}$, i.e. $\Omega=\{T, L, C, D\}$; regarding to data collection fashions, automatic RFID reading and manual recording are two alternative ways. Suppose the number of times the data acquisition has been executed during the maintenance of an EMU was $N_{k}, k \in \Omega$, then total times is $N=\sum_{k \in \Omega} N_{k}$. Average automatic reading time is $\bar{t}_{k}^{A}, k \in \Omega$, while that of manual way is $\bar{t}_{k}{ }^{M}, k \in \Omega$. The error rates of automatic and manual measures are $e_{k}^{A}, k \in \Omega$ and $e_{k}^{M}, k \in \Omega$ respectively. In the view of application logs, we come to a conclusion that failure rate is far less than success rate whatever the data collection mode is; so the time on fail reading $\approx 2 \bar{t}$. Therefore, the increased rate of data acquisition efficiency owing to RFID is:

$$
B=1-\frac{\sum_{k \in \Omega} N_{k} \bar{t}_{k}^{A}+\sum_{k \in \Omega} e_{k}^{A} N_{k} \bar{t}_{k}^{A}}{\sum_{k \in \Omega} N_{k} \bar{t}_{k}^{M}+\sum_{k \in \Omega} e_{k}^{M} N_{k} \bar{t}_{k}^{M}}
$$

Regarding as an example the software logging from 8group type I EMU maintenance at GuangZhou EMU base, we get the following statistics as shown in TABLE I:

TABLE I. SOFTWARE LOGGING STATISTICS OF CRH1 8-GROUP TRAINS MAINTENANCE

\begin{tabular}{|c|c|c|c|c|c|c|c|c|}
\hline & \multicolumn{2}{|c|}{$\begin{array}{l}\text { Trains } \\
\text { (T) }\end{array}$} & \multicolumn{2}{|c|}{$\begin{array}{l}\text { Logistics } \\
\text { (L) }\end{array}$} & \multicolumn{2}{|c|}{ Labor jobs (C) } & \multicolumn{2}{|c|}{$\begin{array}{l}\text { Device assets } \\
\text { (D) }\end{array}$} \\
\hline & \multicolumn{2}{|c|}{$\approx 10$} & \multicolumn{2}{|c|}{$\approx 1700$} & \multicolumn{2}{|c|}{$\approx 250$} & \multicolumn{2}{|c|}{$\approx 60$} \\
\hline $\begin{array}{c}\text { reading time } \\
(\bar{t})\end{array}$ & $<0.1$ & $\approx 10$ & $<0.1$ & $\approx 10$ & $<0.5$ & $\approx 5$ & $<0.5$ & $\approx 10$ \\
\hline $\begin{array}{l}\text { Reading } \\
\text { failure } \\
\text { rate } \\
\text { (e) }\end{array}$ & $0.09 \%$ & $0.57 \%$ & $1.32 \%$ & $1.94 \%$ & $0.08 \%$ & $1.35 \%$ & $0.17 \%$ & $1.84 \%$ \\
\hline
\end{tabular}

$N_{\mathrm{T}}$ is data acquisition times of train identification during its parking and transferring among park lines, test lines and jack-up lines; $N_{L}$ is data acquisition times of logistics identification during examined parts and spare (interchangeable) parts moving within workshops, whose amount is related to EMU static architecture and maintenance process(about 107 car-body processes and 44 running-gear processes); $N_{C}$ is the amount of manual logging in by staffs, whose number is related to group crew and work station terminals; $N_{D}$ is data acquisition times of device identification while equipment or facility maintaining, whose number is related to management specification of spot check, inspection, malfunction report. Compared with RFID, Manual operation needs extra actions like moving, identifying, recording, reporting and etc. According to RFID technical specifications and practical experience, a set of conservative estimates $\left(\bar{t}^{A}, \bar{t}^{M}\right)$ were made. Reading failure rates $e^{A}$ and $e^{M}$ come from the statistical result of software logging, regarding the actions of repeated reading as failure whether through tags or cards. 
Given that failure rates e are tiny relatively, while under various application mode $\mathrm{k}(k \in \Omega), \bar{t}_{\mathrm{k}}^{A} / \bar{t}_{\mathrm{k}}^{M}$ is negligible; the above equation can be simplified to $B=1-\bar{t}^{A} / \bar{t}^{M}$. Assuming $\alpha$ as the workload proportion of data collection to all, therefore the productive improvement promoted by RFID is $S=\alpha B$. Because of $B \approx 1$, then $S \approx \alpha$. After the application of RFID in GuangZhou EMU maintenance base, total productive efficiency increased by $20 \%$; this analytical results is in accordance with practical observation. The above evaluation only concerns with the productive efficiency promotion owing to RFID; however, for the further advantages benefit from the richness and completeness of productive data, as well as later comprehensive information management, IoTsystem has inestimable value.

\section{CONCLUSION}

Based on AEI and RFID, IoT technologies have been widely used in the train dispatching and labor jobs management in GuangZhou EMU maintenance base. Technological solutions for the production-line and spare parts delivery have experienced pilot test with corresponding software like projects scheduling, process control, working processes monitoring, logistics management, etc. Engineering practice showed that IoT technologies benefit the efficiency upgrading of maintenance jobs flow control, the reducing of labor intensity and failure probability, especially the richness of productive information. Further steps will be made for attaining mature application through pilot test and technological solution improvement, while realizing comprehensive utilization of the real-time multi-source productive data of EMU base, establishing an intelligent architecture for EMU maintenance application.

\section{REFERENCES}

[1] Chang Y.S, Oh C.H, Whang Y.S, et al. Development of RFID Enabled Aircraft Maintenance System[C], 2006 IEEE International Conference, 2006(8):224-229.

[2] WANG Zhi-hua, SHI Tian-yun. The Current Application situation of Radio Frequency Identification(RFID) in Transportation [J]. Journal of Transportation Systems Engineering and Information Technology,2005,5(6):96-99.

[3] Weinstein, R.: RFID: A Technical Overview and Its Application to the Enterprise. IT Professional 7.3, (2005).

[4] Lichen Zhang ,Jifeng He. A Formal Framework for Aspect-Oriented Specification of Cyber Physical Systems[J].Communications in Computer and Information Science, 2011,206:391-398.

[5] LIU Weining, HUANG Wenlei, SUN Dihua, et al. Design and implementation of discrete manufacturing industry MES based on RFID Technology[J]. Computer Integrated Manufacturing Systems, 2007, 13(10):1886-1890.

[6] Tetsuro T, Tatsuya I, Osamnu N, et al. A proposal on RFID Data Analytics Methods[C]. Internet of Things (IOT), 2010: 1-6

[7] Ilic A,Andersen T, Michahelles F. Increasing Supply-Chain Visibility with Rule-Based RFID Data Analysis[J].Internet Computing, IEEE,2009(2):31-38.

[8] LUCKHAM D. The power of events: an introduction to complex event processing in distributed enterprise systems[M]. Boston, Mass., USA; Addison-Wesley Professional, 2002.

[9] ZANG Chuanzhen, FAN Yushun. Complex event processing of manufacturing enterprises based on smart items[J]. Computer Integrated Manufacturing Systems, 2007, 43(2):22-32

[10] Hameed, B.; Khan, I.; Dürr, F.; Rothermel, K. An RFID Based Consistency Management Framework for Production Monitoring In a Smart Real-Time Factory[C]. Internet of Things (IOT), 2010: 1-8.

[11] Ilic, A., Andersen, T., Michahelles, F.: Increasing Supply-Chain Visibility with Rule-Based RFID Data Analysis. In: IEEE Internet Computing, vol. 13, no. 1, pp. 31-38, (2009).

[12] De, P., Basu, K., Das, S. K.: An Ubiquitous Architectural Framework and Protocol for Object Tracking Using RFID Tags. In: Proc. of the Intl. Conf. on Mobile and Ubiquitous Systems: Networking and Services, (2004).

[13] Floerkemeier, C., Lampe, M.: RFID middleware design: addressing application requirements and RFID constraints. In: sOc-EUSAI '05: Proc. of the 2005 Joint Conf. on Smart Objects and Ambient Intelligence, (2005). 